\title{
Three-Dimensional Vortex Solitons in Self-Defocusing Media
}

\author{
Nikolaos K. Efremidis, ${ }^{1,2}$ Kyriakos Hizanidis, ${ }^{2}$ Boris A. Malomed, ${ }^{3}$ and Paolo Di Trapani ${ }^{4}$ \\ ${ }^{1}$ Department of Applied Mathematics, University of Crete, 71409 Heraklion, Crete, Greece \\ ${ }^{2}$ School of Electrical and Computer Engineering, National Technical University of Athens, Athens 15773, Greece \\ ${ }^{3}$ Department of Interdisciplinary Studies, Faculty of Engineering, Tel Aviv University, Tel Aviv 69978, Israel \\ ${ }^{4}$ Istituto Nazionale per la Fisica della Materia and Department of Physical and Mathematical Sciences, University of Insubria, \\ Via Valleggio 11, 22100 Como, Italy
}

(Received 1 October 2006; published 14 March 2007)

\begin{abstract}
We demonstrate that families of vortex solitons are possible in a bidispersive three-dimensional nonlinear Schrödinger equation. These solutions can be considered as extensions of two-dimensional dark vortex solitons which, along the third dimension, remain localized due to the interplay between dispersion and nonlinearity. Such vortex solitons can be observed in optical media with normal dispersion, normal diffraction, and defocusing nonlinearity.
\end{abstract}

Vortex solitons are self-localized solutions of nonlinear wave equations, which are characterized by a phase singularity at the pivotal point. The phase charge of a simple closed curve surrounding the vortex core is equal to $2 \pi m$, where $m$ is the integer vorticity of the solution. Vortex solitons have been theoretically predicted in the context of superfluids $[1,2]$. In those early works, a two-dimensional (2D) nonlinear Schrödinger (NLS) equation with defocusing nonlinearity was shown to support vortex soliton solutions whose intensity vanishes at the vortex center and asymptotically approaches a constant value at infinity. Such dark vortex solitons were experimentally observed in a bulk self-defocusing optical medium [3]. The stability of nonlinear vortices depends on the vorticity number, $m$. Fundamental vortices with $m=1$ are energetically favorable (and, as a result stable), which implies that instabilities of other families of solutions may result to the formation of (a set of) fundamental vortices. In particular, a 2D dark soliton stripe is unstable to long-wave symmetry breaking perturbations, leading to the generation of fundamental vortex-soliton pairs with opposite vorticities [4-6]. Higher order vortices are also unstable and break down into fundamental vortices. However, in the NLS limit no exponentially growing mode exists [7] (the instability may be subexponential) and, as a result, multicharged vortices are very long-lived objects. Strong instabilities of multicharged vortices can be triggered by different mechanisms such as dissipation, nonlinearity saturation, or anisotropy [8].

Another class of solutions feature ring-shaped intensity profiles and exist in self-focusing media [9]. Such solutions are unstable even in the case of saturable nonlinearity due to azimuthal instabilities and break down to a set of fundamental solitons [10]. Ring vortices can be stabilized if the model includes a combination of competing (selffocusing and self-defocusing) nonlinear terms, such as cubic and quintic $[11,12]$ or quadratic and self-defocusing cubic [13] ones. Azimuthal instabilities can also be sup- pressed by appropriately modulating the amplitude of the solution in the angular direction [14]. Recently a lot of attention has been attracted to the study of vortices in periodic lattices. The lattice can stabilize families of ring vortices [15-19] by trapping the intensity in the lattice potential minima. In a different setting, vortex solitons were observed in Bose-Einstein condensates [20,21].

In contrast to the plethora of theoretical and experimental works on vortices in two dimensions, only a few works have addressed 3D solutions. Stable toroidal solitons with vorticity 1 were found in systems with competing nonlinearities [13,22], and a variety of 3D discrete solitons of the vortex type were constructed and explored in Refs. [23,24]. In addition, propagation and robustness of 3D ring optical vortices in the atmosphere was examined in Ref. [25]. Focusing properties of bidispersive (normal dispersion and normal diffraction) optical systems have been studied in [26,27] for self-focusing nonlinearities.

The subject of the present Letter is to find 3D counterparts of the dark optical vortices which were discovered long ago in Refs. [1,2], and to predict experimental conditions necessary for their observation. We demonstrate that $3 \mathrm{D}$ vortices exist in media with the cubic defocusing nonlinearity and normal group velocity dispersion (GVD), i.e., the diffraction-dispersion operator is of the hyperbolic type. Optical media realizing this model are available, such as specific AlGaAs alloys [28,29]. In the transverse $(x, y)$ plane, these solutions have the form of a dark vortex, whereas along the longitudinal axis (i.e., in the temporal direction) they remain localized as bright temporal solitons, due to the interplay between the normal GVD and defocusing nonlinearity. We first construct the solutions in a semianalytical (and quite accurate) form, making use of the Hartree approximation [30]. Then, we employ the Newton iteration method to find the 3D vortices as numerical solutions to the underlying NLS equation. The Hartree approximation provides the Newton's method with appropriate initial conditions. The stability of the vortices is 
tested by direct numerical simulations. We conclude that $3 \mathrm{D}$ vortices are stable (as long as a super-Gaussian carrying the vortex in the transverse plane, which is as a part of the numerical procedure, does not suffer essential diffraction). Considering applications, the central hole in such a vortex pancake may be used as an optically induced aperture to dynamically control a probe beam passing through it.

We start the analysis by introducing the normalized NLS equation,

$$
i \psi_{z}+\frac{1}{2}\left(\nabla_{\perp}^{2} \psi-\psi_{t t}\right)-|\psi|^{2} \psi=0,
$$

where $\nabla_{\perp}^{2}=\partial_{x}^{2}+\partial_{y}^{2}$ is the diffraction operator, with the normal GVD dispersion and defocusing nonlinearity coefficients scaled to unity. In addition to the aforementioned optical media (AlGaAs alloys), Eq. (1) applies, as the Gross-Pitaevskii equation, to Bose-Einstein condensate in an optical lattice, where $z$ is now time, and $t$ a spatial coordinate across the lattice. In this case, the negative diffraction along $t$ is achieved when the Bloch momentum corresponds to a negative effective mass.

Notice that if $\psi(x, y, z, t)$ is a solution of Eq. (1), then $\psi^{\prime}=\alpha \psi\left(\alpha x, \alpha y, \alpha t, \alpha^{2} z\right)$, with real free parameter $\alpha$, is a solution too. Using this scale-invariance property, a oneparameter family of solutions can be generated from a single solution of Eq. (1). Introducing polar coordinates $(\rho, \phi)$ in the $(x, y)$ plane, we look for solutions of Eq. (1) in the form of

$$
\psi(\rho, \phi, t, z)=u(\rho, t) \exp (-i k z) \exp (i m \phi),
$$

where $m$ is integer vorticity. Substituting Eq. (2) in Eq. (1) and using the above-mentioned scale invariance to set $k=$ $1 / 2$, we obtain

$$
u+\left(\rho^{-1} u_{\rho}+u_{\rho \rho}-m^{2} \rho^{-2} u\right)-u_{t t}-2 u^{3}=0 .
$$

The asymptotic expansion of the $t$-independent problem at $\rho \rightarrow \infty$ yields $u=(1 / \sqrt{2})\left[1-\left(m^{2} / 2\right) \rho^{-2}-\left(m^{2} / 8\right) \times\right.$ $\left.\left(8+m^{2}\right) \rho^{-4}\right]+\mathcal{O}\left(\rho^{-6}\right)$. On the other hand, the asymptotic expansion at $\rho \rightarrow 0$ is $u=c\left\{\rho^{|m|}-[1 /(4(m+\right.$ 1) $\left.] \rho^{|m|+2}\right\}+\mathcal{O}\left(\rho^{|m|+4}\right)$. Accordingly, we look for solutions of the time-dependent problem with $\lim _{\rho \rightarrow 0} u(\rho, t)=$ 0 and $\lim _{\rho \rightarrow \infty} u(\rho, t)=u_{\infty}(t)$. In the latter limit, Eq. (3) reduces to the dynamical system associated with the single-soliton solution of the NLS equation, $u_{\infty}-u_{\infty, t t}-$ $2 u_{\infty}^{3}=0$, whose commonly known soliton solution is

$$
\lim _{\rho \rightarrow \infty} u(\rho, t)=u_{\infty}(t)=\operatorname{sech}(t),
$$

in compliance with our objective to find solutions that look as bright solitons in the temporal direction.

The total energy conserved by Eq. (1) is

$$
P=\int_{-\infty}^{\infty} \int_{-\infty}^{\infty} \int_{-\infty}^{\infty}|\psi(x, y, t)|^{2} d x d y d t .
$$

However, this integral of motion diverges for solutions with asymptotic form (4). A renormalized (convergent) form of the energy, that takes into account the asymptotic form of the solution, may be defined as

$$
P_{n}=\int_{-\infty}^{\infty} \int_{-\infty}^{\infty} \int_{-\infty}^{\infty}\left[u_{\infty}^{2}(t)-|\psi|^{2}\right] d x d y d t
$$

Notice that $\psi_{s}=u_{\infty}(t) e^{i z / 2}=\operatorname{sech}(t) e^{i z / 2}$ is a solution of Eq. (1) representing a bright soliton stripe in three dimensions. As a result, both terms in Eq. (6) obey the abovementioned scale invariance, which can be used to derive a rule for generating a one-parameter family of vortex solitons from a single one: $P_{n}(\alpha)=P_{n}(1) / \alpha$.

We start the analysis by resorting to the Hartree approximation (HA), which is based on the product ansatz [30],

$$
u(\rho, t)=R(\rho) T(t) .
$$

This approximation will then be used as an initial guess for numerical solutions based on the Newton's method. Substituting Eq. (7) in Eq. (3), we arrive at a formal equation,

$$
\left(\rho^{-1} R^{\prime}+R^{\prime \prime}-m^{2} \rho^{-2} R+R\right) T-R T^{\prime \prime}-R^{3} T^{3}=0 .
$$

First, Eq. (8) is to be multiplied with $R$, integrated over $\rho$ from 0 to $\rho_{1}$, and divided by $\int_{0}^{\rho_{1}} R^{2} d \rho$. Taking the limit of $\rho_{1} \rightarrow \infty$, the equation

$$
T-T^{\prime \prime}-2 T^{3}=0 .
$$

is derived by assuming (without loss of generality) that $R(\rho \rightarrow \infty)=1$. A relevant solution to Eq. (9) is the same temporal bright soliton as the one obtained above as the asymptotic wave form, $T(t)=\operatorname{sech}(t)$. Next, we multiply Eq. (8) by $T$, integrate it from $-t_{1}$ to $t_{2}$, divide by $\int_{-t_{1}}^{t_{2}} T^{2}(t) d t$, and take the limits of $t_{1} \rightarrow \infty, t_{2} \rightarrow \infty$, to obtain

$$
3\left(\rho^{-1} R^{\prime}+R^{\prime \prime}-m^{2} \rho^{-2} R\right)+4\left(R-R^{3}\right)=0 .
$$

Equation (10) can be solved numerically by dint of standard two-point boundary-value methods, such as shooting.

All our direct numerical simulations are performed using the beam propagation method, where the linear part is solved using the fast Fourier transform and the nonlinear part by direct integration (see, for example, [31]). Since the solution does not vanish at infinity, and aiming to avoid artifacts produced by reflections from domain boundaries, we took a 12th order super-Gaussian (in $\rho$ ) as a finiteextension carrier for the solution. Of course, diffraction of the super-Gaussian cannot be avoided, hence, after a large but finite propagation distance, the field starts to decay. Eventually, this leads to destabilization of the vortex.

In Fig. 1 the evolution of an initial configuration suggested by the HA is depicted. Notice that the simulation window is truncated for large values of $\rho$ so as to display only the evolution of the vortex soliton while eliminating the above-mentioned irrelevant effect of the background 

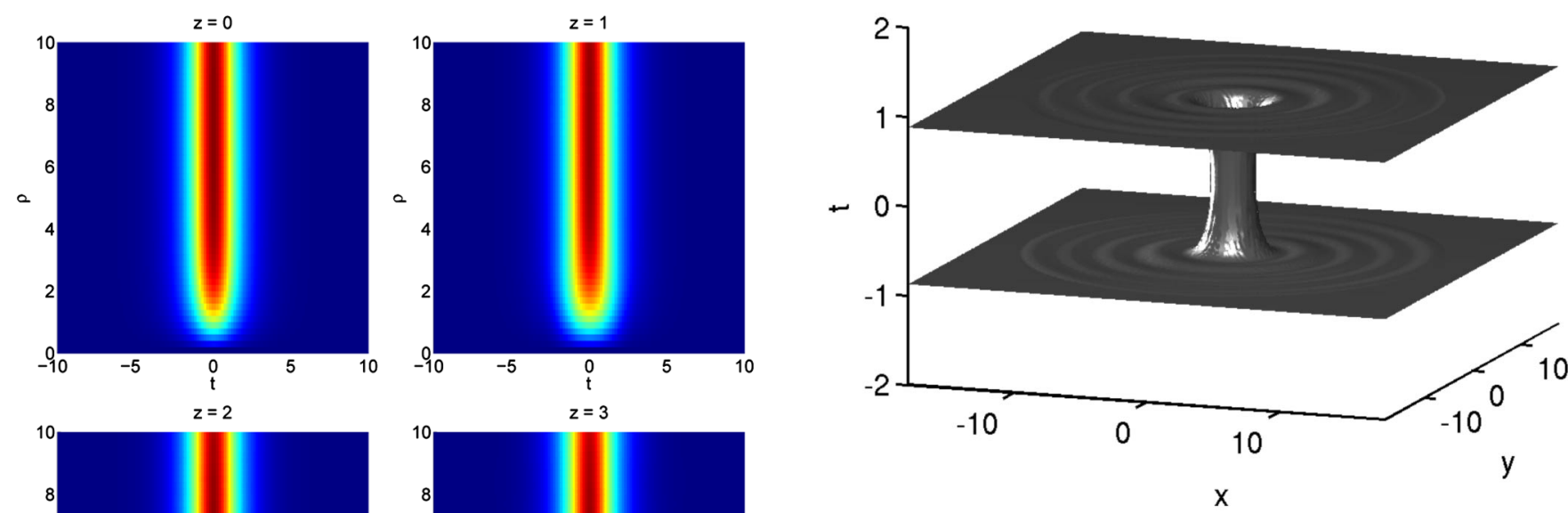

FIG. 2. Isosurface plot, corresponding to $|u|^{2}=0.5$, of the fundamental vortex-soliton solution obtained by means of the Newton iteration method.

showed similar evolution initiated by the HA, attests to the accuracy of that approximation. A noteworthy feature revealed by Fig. 3 (in a more salient form than by the HA cf. Fig. 1) is that, close to the vortex core (at small values of $\rho$ ), the vortex is wider in the $t$ direction. We stress that the vortical phase structure of the solution is maintained during the propagation.

To directly verify that such a $3 \mathrm{D}$ vortex-soliton solution

diffraction. In all simulations, the initial conditions did not develop azimuthal instabilities, i.e., the intensity distribution corresponding to the vortex soliton did not generate any dependence on $\phi$, nor did it develop any other instability. Furthermore, as the vortex propagates, it slightly broadens in $t$ close to its center. This effect does not imply any trend to decay of the vortex. It is, rather, a manifestation of the relaxation of the initial approximate wave form towards an exact vortex state. As shown below, this conclusion is in agreement with results generated by the Newton iteration method.

To generate numerically exact stationary vortex-soliton solutions, the HA was fed, as an initial guess, into the Newton's method for Eq. (3). Notice that the discretization of Eq. (3) in variable $\rho$ requires special attention, since the error is $\rho$-dependent. More specifically, the discretization error becomes larger as $\rho$ decreases, whereas at large values of $\rho$ the error is almost isotropic (independent of $\rho$, to the leading order). The iteration provided for the convergence of solutions fed by the above-mentioned initial HA configurations. The isointensity profile of the so obtained fundamental vortex soliton is depicted in Fig. 2.

In Fig. 3, direct simulations of the propagation of a 3D vortex soliton, with the initial condition generated by the Newton's iteration method, is shown (as above, the initial configuration was actually multiplied by the 12th order super-Gaussian). Notice that the vortex-soliton profile remains invariant in the course of the propagation, i.e., the vortex soliton is stable. Comparison with Fig. 1, which is a nonlinear object indeed, we repeated the same simulations, dropping the nonlinear term. As one can see in Fig. 4, the vortex quickly diffracts in that case by at $z=3$.
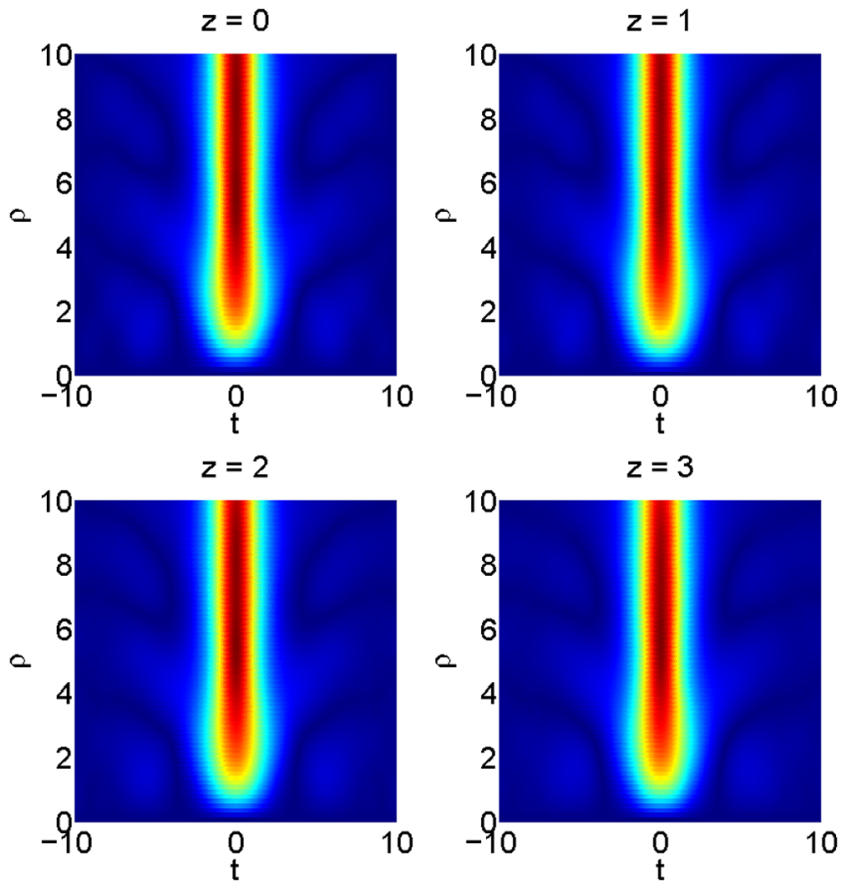

FIG. 3 (color online). The same as in Fig. 1, but if the initial condition was taken as a stationary solution generated by the Newton's iteration method. 

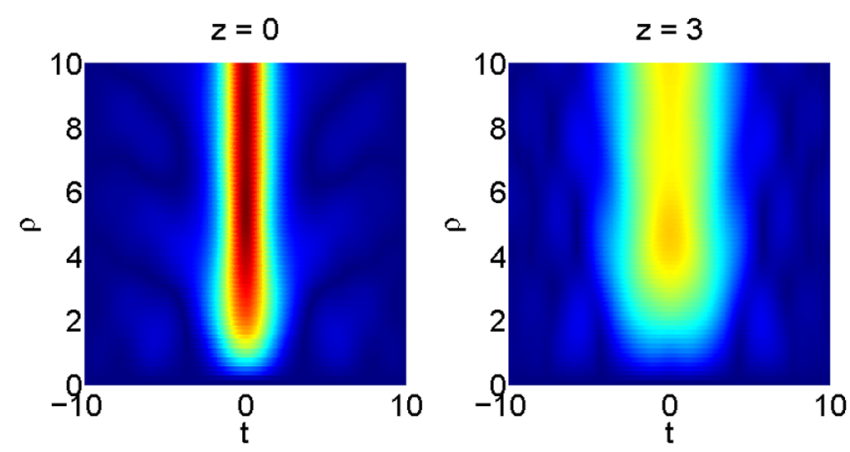

FIG. 4 (color online). Propagation of a fundamental $(m=1)$ vortex soliton up to $z=0,3$ at which point the nonlinearity is switched off.

We have also performed simulations for double vortices, with $m=2$. They were found to propagate undistorted over large distances, without splitting into fundamental vortices. This observation is, in fact, in agreement with a conjecture put forward in Ref. [7].

In conclusion, we have demonstrated that vortex solitons are possible in the bidispersive 3D NLS equation. These solutions have the form of a dark vortex in the spatial plane, whereas they are localized along the temporal dimension, due to the interplay between the GVD and nonlinearity. Such vortex solitons can be observed in certain optical media with normal GVD, normal diffraction, and defocusing nonlinearity, and in self-repulsive BoseEinstein condensation.

N.K.E. and K.H. Acknowledge funding by the European Social Fund (75\%) and National Resources (25\%)-Operational Program for Educational and Vocational Training II (EPEAEK II) and particularly the Program PYTHAGORAS. B. A. M. appreciates hospitality of the National Technical University of Athens.

[1] V. Ginzburg and L. Pitaevskii, Zh. Eksp. Teor. Fiz. 34, 1240 (1958) [Sov. Phys. JETP 7, 858 (1958)].

[2] L. Pitaevskii, Zh. Eksp. Teor. Fiz. 40, 646 (1961) [Sov. Phys. JETP 13, 451 (1961)].

[3] G. Swartzlander, Jr. and C. Law, Phys. Rev. Lett. 69, 2503 (1992).

[4] C. Law and G. A. Swartzlander, Jr., Opt. Lett. 18, 586 (1993).

[5] A. Mamaev, M. Saffman, and A. Zozulya, Phys. Rev. Lett. 76, 2262 (1996).

[6] V. Tikhonenko, J. Christou, B. Luther-Davies, and Y.S. Kivshar, Opt. Lett. 21, 1129 (1996).
[7] I. Aranson and V. Steinberg, Phys. Rev. B 53, 75 (1996).

[8] A. Mamaev, M. Saffman, and A. Zozulya, Phys. Rev. Lett. 78, 2108 (1997).

[9] V. Kruglov and R. Vlasov, Phys. Lett. A 111, 401 (1985).

[10] V. Tikhonenko, J. Christou, and B. Luther-Davies, J. Opt. Soc. Am. B 12, 2046 (1995).

[11] L.-C. Crasovan, B. Malomed, and D. Mihalache, Pramana 57, 1041 (2001).

[12] M. Quiroga-Teixeiro and H. Michinel, J. Opt. Soc. Am. B 14, 2004 (1997).

[13] D. Mihalache, D. Mazilu, L.-C. Crasovan, I. Towers, B. Malomed, A. Buryak, L. Torner, and F. Lederer, Phys. Rev. E 66, 016613 (2002).

[14] A. S. Desyatnikov, A. A. Sukhorukov, and Y.S. Kivshar, Phys. Rev. Lett. 95, 203904 (2005).

[15] V. Baizakov, B. Malomed, and M. Salerno, Europhys. Lett. 63, 642 (2003).

[16] J. W. Fleischer, G. Bartal, O. Cohen, O. Manela, M. Segev, J. Hudock, and D. N. Christodoulides, Phys. Rev. Lett. 92, 123904 (2004).

[17] B. A. Malomed and P. G. Kevrekidis, Phys. Rev. E 64, 026601 (2001).

[18] D. N. Neshev, T. J. Alexander, E. A. Ostrovskaya, Y.S. Kivshar, H. Martin, I. Makasyuk, and Z. Chen, Phys. Rev. Lett. 92, 123903 (2004).

[19] J. Yang and Z.H. Musslimani, Opt. Lett. 21, 2094 (2003).

[20] B. P. Anderson, P. C. Haljan, C. A. Regal, D. L. Feder, L. A. Collins, C. Clark, and E. A. Cornell, Phys. Rev. Lett. 86, 2926 (2001).

[21] N. S. Ginsberg, J. Brand, and L. V. Hau, Phys. Rev. Lett. 94, 040403 (2005).

[22] D. Mihalache, D. Mazilu, L.-C. Crasovan, I. Towers, A. Buryak, B. A. Malomed, L. Torner, J. P. Torres, and F. Lederer, Phys. Rev. Lett. 88, 073902 (2002).

[23] R. Carretero-González, P. Kevrekidis, B. Malomed, and D. Frantzeskakis, Phys. Rev. Lett. 94, 203901 (2005).

[24] P. Kevrekidis, B. Malomed, D. Frantzeskakis, and R. R. Carretero-González, Phys. Rev. Lett. 93, 080403 (2004).

[25] A. Vinçotte and L. Bergé, Phys. Rev. Lett. 95, 193901 (2005).

[26] L. Bérge, E. A. Kuznetsov, and J. J. Rasmussen, Phys. Rev. E 53, R1340 (1996).

[27] L. Bérge, J. J. Rasmussen, E. A. Kuznetsov, E. G. Shapiro, and S. K. Turitsyn, J. Opt. Soc. Am. B 13, 1879 (1996).

[28] P. Dumais, A. Villeneuve, and J. S. Aitchison, Opt. Lett. 21, 260 (1996).

[29] S.-P. Gorza, N. Roig, P. Emplit, and M. Haelterman, Phys. Rev. Lett. 92, 084101 (2004).

[30] K. Hayata and M. Koshiba, Phys. Rev. E 48, 2312 (1993).

[31] A.C. Newell and J. V. Moloney, Nonlinear Optics (Addison-Wesley, Redwood City, CA, 1992). 